\title{
Holographic Cosmology from the First Law of Thermodynamics and the Generalized Uncertainty Principle
}

\author{
James E. Lidsey \\ Astronomy Unit, School of Mathematical Sciences, \\ Queen Mary University of London, Mile End Road, London, E1 4NS, UK
}

\begin{abstract}
The cosmological Friedmann equation sourced by the trace anomaly of a conformal field theory that is dual to the five-dimensional Schwarzschild-AdS geometry can be derived from the first law of thermodynamics if the apparent horizon of the boundary spacetime acquires a logarithmicallycorrected Bekenstein-Hawking entropy. It is shown that such a correction to the entropy can arise when the generalized uncertainty principle (GUP) is invoked. The necessary condition for such a thermodynamic derivation directly relates the GUP parameter to the conformal anomaly. It is consistent with the existence of a gravitational cutoff at a scale $l_{\mathrm{uv}} \gtrsim \sqrt{n} l 4$ for a theory containing $n$ light species. The absolute minimum in position uncertainty can be identified with the scale at which gravity becomes effectively five-dimensional.
\end{abstract}

PACS numbers: $98.80 . \mathrm{Cq}$

A large number of matter fields are predicted to exist in unified field theories such as string theory. In the high energy environment of the early universe, such fields should behave as a conformal field theory (CFT). Oneloop quantum corrections break the conformal invariance of the fields and generate a Weyl (trace) anomaly in the energy-momentum tensor of the CFT. In general, this is given by

$$
g^{\mu \nu}\left\langle T_{\mu \nu}\right\rangle=c I_{(4)}-b E_{(4)},
$$

where $I_{(4)}=C_{\mu \nu \lambda \kappa} C^{\mu \nu \lambda \kappa}$ is the square of the Weyl tensor and $E_{(4)}=R^{2}-4 R_{\mu \nu} R^{\mu \nu}+R^{\mu \nu \lambda \kappa} R_{\mu \nu \lambda \kappa}$ is the GaussBonnet invariant. (For a review, see, e.g., Ref. [1].) The field content of the CFT determines the numerical values of the coefficients:

$$
\begin{aligned}
b & =\frac{1}{360(4 \pi)^{2}}\left(n_{0}+11 n_{1 / 2}+62 n_{1}\right) \\
c & =\frac{1}{120(4 \pi)^{2}}\left(n_{0}+6 n_{1 / 2}+12 n_{1}\right),
\end{aligned}
$$

where $n_{0}, n_{1 / 2}$ and $n_{1}$ are the number of scalar, Dirac fermion and vector fields, respectively.

Conformal field theories have a holographic spacetime dual in the large $n$ limit if $b=c$ [2]. For example, the AdS/CFT correspondence implies that $\mathcal{N}=4 S U(N)$ super-Yang-Mills theory is dual to type IIB string theory on $\mathrm{AdS}_{5} \times \mathrm{S}^{5}[3-6]$. More generally, the properties of the CFT are determined by the geometry of the dual spacetime. In particular, the energy-momentum tensor of the CFT is determined by means of a holographic renormalization scheme [2, 7, 8]. For a given solution $G$ to the five-dimensional Einstein field equations sourced by a negative cosmological constant, $\Lambda_{5}$, the metric is written in the form $d s_{5}^{2}=\ell^{2} z^{-2}\left[d z^{2}+g_{\mu \nu} d x^{\mu} d x^{\nu}\right]$, where

$$
g_{\mu \nu}=g_{\mu \nu}^{(0)}+g_{\mu \nu}^{(2)} \frac{z^{2}}{\ell^{2}}+g_{\mu \nu}^{(4)} \frac{z^{4}}{\ell^{4}}+\ldots,
$$

and $g_{\mu \nu}^{(i)}=g_{\mu \nu}^{(i)}(x)$ solve the gravitational field equations. The $z$-coordinate is chosen so that the boundary of $G$ is represented by $z=0$. It can then be shown that the holographic conformal anomaly is given by $[2,7,8]$

$$
\left\langle g^{(0) \mu \nu} T_{\mu \nu}^{(\text {holo })}\right\rangle=\frac{\ell^{3}}{128 \pi l_{5}^{3}}\left(I_{(4)}-E_{(4)}\right),
$$

where $\ell$ is the curvature radius of $\operatorname{AdS}_{5}$ and $l_{5}$ is the five-dimensional Planck length. Eq. (44) corresponds to the standard, four-dimensional, field-theoretic result (1) with $b=c=\ell^{3} /\left(128 \pi l_{5}^{3}\right)$.

The cosmological consequences of such a gauge theory/gravity duality can be investigated by parametrizing the line-element (3) so that the boundary metric $g_{\mu \nu}^{(0)}$ takes the spatially flat Friedmann-RobertsonWalker (FRW) form. This metric is made dynamical when appropriate mixed boundary conditions are imposed and a boundary Einstein action is introduced in the holographic renormalization [9]. The effective fourdimensional field equations are then given by $R_{\mu \nu}-$ $\frac{1}{2} R g_{\mu \nu}^{(0)}=8 \pi l 4^{2}\left\langle T_{\mu \nu}^{(\text {holo })}\right\rangle$, where $l 4$ denotes the fourdimensional Planck length. Recently, it was shown that when the gravity dual is the Schwarzschild- $\mathrm{AdS}_{5}$ geometry, the (00)-component of the Einstein equations takes the form [10]

$$
H^{2}-16 \pi b l 4^{2} H^{4}=\frac{8 \pi l 4^{2}}{3} \rho,
$$

where $\rho=\mathcal{C} / a^{4}$ may be interpreted as the energy density of a conformally invariant classical fluid, the constant $\mathcal{C}$ is determined by the mass of the bulk black hole, $H \equiv \dot{a} / a$ and $a(t)$ denotes the scale factor.

Eq. (5) can also be derived directly from the trace anomaly (11) for a generic CFT (with $b \neq c$ ) by integrating the contracted Bianchi identity [1]. In this case, the parameter $\mathcal{C}$ arises as the arbitrary integration constant.

The purpose of the present paper is to show that the holographic/conformal-anomaly Friedmann equation (5) admits an alternative derivation in terms of spacetime thermodynamics and the generalized uncertainty prin- 
ciple of quantum gravity. It has long been appreciated that a deep connection exists between gravitation, quantum theory and statistical physics 12 17]. One of the central themes underlying this connection is that a spacetime horizon should be associated with an entropy that is directly proportional to the horizon area [12, 17]. When such a proportionality exists for a local Rindler causal horizon, the Einstein field equations can be derived from the fundamental Clausius relation [18]. More specifically, the standard Friedmann equations for a spatially isotropic universe follow directly from the first law of thermodynamics if the entropy and area of the apparent horizon satisfy the Bekenstein-Hawking formula, $S=A /\left(4 l 4^{2}\right)[19]$.

Here we consider the effect of the generalized uncertainty principle (GUP) on the apparent horizon entropy. The GUP is formulated as the condition

$$
\Delta x \Delta p \gtrsim 1+\alpha^{2} l 4^{2}(\Delta p)^{2},
$$

where $\alpha$ is a (model-dependent) dimensionless constant. Such a modification to the standard Heisenberg relation has been derived in a number of different approaches to quantum gravity, including non-commutative quantum mechanics [20] and string theory [21]. It also arises from Gedanken experiments that are independent of the underlying theory [22]. (For a review, see, e.g., Ref. [23].) We find that the GUP induces a logarithmic correction to the entropy that has precisely the form required for a thermodynamic derivation of the Friedmann equation (5).

To proceed, we adapt a line of reasoning developed within the context of black hole spacetimes [24]. The spatially flat FRW line element can be written in the form $d s^{2}=h_{a b} d x^{a} d x^{b}+\tilde{r}^{2} d \Omega_{2}^{2}$, where the two-metric $h_{a b}=\operatorname{diag}\left(-1, a^{2}\right)$ and $\tilde{r} \equiv r a(t)$. The apparent horizon of an observer at $r=0$ is the constant-time hypersurface where orthogonal ingoing, future-directed light rays have zero expansion. It corresponds to a sphere of radius $\tilde{r}_{A}=$ $1 / H$ and area $A=4 \pi \tilde{r}_{A}^{2}$, where $\tilde{r}_{A}$ is defined by the condition $h^{a b} \tilde{r}_{, a} \tilde{r}_{, b}=0$.

In the following, we focus on the regime of cosmic dynamics where the universe undergoes a phase of quasiexponential expansion, such that $\dot{\tilde{r}}_{A}=-\dot{H} / H^{2} \ll 1$. This implies that over the incremental time intervals considered, the apparent horizon radius can be regarded as having a fixed value. (Such inflationary expansion can be realised by introducing an effective four-dimensional cosmological constant that is generated by a slowly rolling, self-interacting scalar field. We do not exhibit such a term in the Friedmann equations for notational simplicity.)

Suppose the apparent horizon absorbs (or emits) a massless quantum particle of energy, $\Delta E$. This is determined by the corresponding uncertainty in the particle's momentum, $\Delta E \simeq \Delta p$ [25]. The effective massenergy within the horizon will then change by an amount $d \mathcal{M} \simeq \Delta p$. The total mass-energy within the apparent horizon is given by $\mathcal{M}=4 \pi \tilde{r}_{A}^{3} \rho / 3$ and this can be expressed in the form $\mathcal{M}=\tilde{r}_{A} /\left(2 l 4^{2}\right)$ after substitution of the Friedmann equation, $H^{2}=8 \pi l 4^{2} \rho / 3$. (More generally, the horizon mass is the Misner-Sharp mass $\mathcal{M} \equiv \tilde{r}\left[1-h^{a b} \tilde{r}_{, a} \tilde{r}_{, b}\right] /\left(2 l 4^{2}\right)$ evaluated at the radius $\tilde{r}=\tilde{r}_{A}[26]$.) The corresponding change in the horizon area as a result of the absorption is therefore

$$
\Delta A \simeq 16 \pi l 4^{2} \tilde{r}_{A} \Delta p .
$$

The particle will have a Compton wavelength and associated uncertainty in position, $\Delta x$. A natural length scale for this uncertainty is the inverse of the surface gravity at the apparent horizon. This is given by $|\kappa|^{-1}=$ $\tilde{r}_{A}\left[1-\dot{\tilde{r}}_{A} / 2\right]^{-1} \simeq \tilde{r}_{A}$. Hence, the position uncertainty can be estimated as

$$
\Delta x \simeq \tilde{r}_{A} .
$$

In this case, it follows that the change in horizon area is $\Delta A \simeq\left(16 \pi l 4^{2}\right) \Delta x \Delta p$. The standard form of the Heisenberg uncertainty principle, $\Delta x \Delta p \gtrsim 1$, would then impose a lower bound of $\Delta A \gtrsim 16 \pi l 4^{2}$. However, it is straightforward to show that the GUP results in a lower bound on the momentum uncertainty (for a given value of $\Delta x$ ):

$$
\Delta p \gtrsim \frac{\Delta x}{2 \alpha^{2} l 4^{2}}\left[1-\sqrt{1-\frac{4 \alpha^{2} l 4^{2}}{(\Delta x)^{2}}}\right] .
$$

We then deduce that

$$
\Delta A \gtrsim \frac{2 A}{\alpha^{2}}\left[1-\sqrt{1-\frac{16 \pi \alpha^{2} l 4^{2}}{A}}\right]
$$

after noting that the area of the apparent horizon is $A \simeq$ $4 \pi(\Delta x)^{2}$.

If we further assume that the energy scales of interest are sufficiently small, $l 4^{2} / A \simeq H^{2} l 4^{2} \ll 1$, to allow for a consistent Taylor expansion of the square root, the bound (10) approximates at leading order to

$$
\Delta A \gtrsim 16 \pi l 4^{2}\left[1+\frac{4 \pi \alpha^{2} l 4^{2}}{A}+\ldots\right] .
$$

This enables us to express the minimum change in the area of the apparent horizon as

$$
(\Delta A)_{\min } \simeq \epsilon l 4^{2}\left[1+\frac{4 \pi \alpha^{2} l 4^{2}}{A}+\ldots\right],
$$

where $\epsilon \simeq \mathcal{O}(16 \pi)$ quantifies any further uncertainties that may arise [24].

The absorption (or emission) of the particle by the horizon results in an increase in the entropy, $\Delta S$. Information theory implies that the minimal increase should be one 'bit of information', $(\Delta S)_{\min } \simeq b$, where $b \in \Re^{+}$ and is independent of the area. (For a review, see, e.g., Ref. 27].) It follows, therefore, that

$$
\frac{(\Delta S)_{\min }}{(\Delta A)_{\min }} \simeq \frac{d S}{d A} \simeq \frac{b}{\epsilon l 4^{2}}\left[1-\frac{4 \pi \alpha^{2} l 4^{2}}{A}+\ldots\right]
$$


and integration of Eq. (13) then yields

$$
S=\frac{A}{4 l 4^{2}}-\pi \alpha^{2} \ln \left(\frac{A}{4 l 4^{2}}\right)+\ldots,
$$

where we have normalized $b / \epsilon=1 / 4$ to reproduce the Bekenstein-Hawking formula in the limit $\alpha^{2} \rightarrow 0$ [24]. In the context of the present discussion, this is equivalent to requiring that the standard, classical Friedmann equation is recovered in the low-energy limit.

To summarize thus far, the effect of the generalized uncertainty principle on the entropy of the apparent horizon is to induce a leading-order logarithmic correction to the Bekenstein-Hawking formula.

We now apply the first law of thermodynamics, $d E=$ $-T d S$, to the apparent horizon. For a universe sourced by a perfect fluid with energy density, $\rho$, and pressure, $P$, the amount of energy, $d E$, crossing the apparent horizon in an infinitesimal time interval, $d t$, is evaluated by integrating the energy-momentum flux through the horizon and contracting with the horizon generator, $k^{a}=(1,-H r)$. It can then be shown that

$$
d E=-A T_{a b} k^{a} k^{b} d t=\frac{4 \pi}{3 H^{3}} d \rho,
$$

where it has been assumed implicitly that the fluid satisfies the conservation equation, $\dot{\rho}=-3 H(\rho+P)$.

If the apparent horizon has an entropy, $S$, and associated Hawking temperature, $T=H /(2 \pi)$ [28], it follows that the first law of thermodynamics can be expressed in the form

$$
d S=-\frac{8 \pi^{2}}{3} \frac{d \rho}{H^{4}} .
$$

After substitution of the logarithmically-corrected Bekenstein-Hawking entropy (14), where $A=4 \pi / H^{2}$, Eq. (16) may be integrated to yield [29, 30]

$$
H^{2}-\frac{\alpha^{2} l 4^{2}}{2} H^{4}=\frac{8 \pi l 4^{2}}{3} \rho .
$$

The effect of the logarithmic correction to the entropy is to modify the Friedmann equation from its standard, relativistic form. A direct correspondence between the holographic/trace-anomaly Friedmann equation (5) and the thermodynamic Friedmann equation (17) is established if the GUP parameter, $\alpha$, and the anomaly coefficient, $b$, are related such that

$$
\alpha^{2}=32 \pi b=\frac{1}{4} \frac{\ell^{3}}{l_{5}^{3}} .
$$

The first equality in the correspondence (18) holds for a generic CFT, irrespective of any holographic considerations, whereas the second arises when there exists a spacetime dual to the CFT (i.e., when $b=c$ ). In a sense, Eq. (18) may be regarded as a triality between a thermodynamic quantity, $\alpha$, a field-theoretic parameter, $b$, and the higher-dimensional gravitational coupling, $l_{5}$.
A question that naturally arises is whether such a correspondence is more than an intriguing mathematical analogy. It is known that logarithmic corrections to the entropy-area law generically arise for black hole spacetimes when quantum effects are taken into account. Indeed, one-loop effects near the event horizon of a Schwarzschild black hole lead to a similar proportionality between the logarithmic coefficient and the conformal anomaly [31]. This suggests that in both the black hole and cosmological environments, the conformal anomaly which is geometric in nature and quantum-mechanical in origin - may be interpreted in terms of a thermodynamic quantity.

We may gain further insight by noting that an immediate consequence of the GUP is that the second term in (6) results in an absolute minimum in the uncertainty in position for any level of momentum uncertainty:

$$
(\Delta x)_{\min } \gtrsim 2|\alpha| l 4 .
$$

Moreover, a generic feature of a theory containing $n$ massless fields coupled to gravity is the existence of a fundamental length scale, $l_{\mathrm{uv}}$, below which low-energy perturbation theory is expected to break down. Since the GUP originates from quantum gravity considerations, it is natural to associate the minimum length scale with this ultraviolet (UV) cutoff. A conservative estimate is that the cutoff occurs at, or on a scale slightly above, $(\Delta x)_{\min }$, i.e., $l_{\mathrm{uv}} \gtrsim(\Delta x)_{\min } \gtrsim 2|\alpha| l 4$. In this case, Eq. (18) implies that

$$
b \lesssim \frac{1}{128 \pi} \frac{l_{\mathrm{uv}}^{2}}{l 4^{2}} .
$$

On the other hand, the number of light species in a typical Grand Unified Theory is roughly the number of gauge bosons. It then follows from Eq. (21) that $b \gtrsim n /\left(100 \pi^{2}\right)$ and substituting this relation into Eq. (20) leads to the condition

$$
l_{\mathrm{uv}} \gtrsim \sqrt{n} l 4 .
$$

This bound for the cutoff is in agreement with independent perturbative [32] and non-perturbative [33, 34] analyses.

Eq. (18) also provides a way of quantifying the model-dependent GUP parameter (and consequently the four-dimensional UV cutoff) directly in terms of fivedimensional length scales. This is interesting from the holographic perspective, since the strong coupling scale in four dimensions is determined by the characteristic scale of the higher-dimensional gravity [34, 35]. For $\mathrm{AdS}_{5}$, one would therefore expect that Eq. (18) should be consistent with the condition $l_{\mathrm{uv}} \simeq \ell$. To verify this, we substitute $l_{\mathrm{uv}} \simeq 2|\alpha| l 4$ into Eq. (18) to deduce that

$$
\frac{l_{\mathrm{uv}}^{2}}{l 4^{2}}=\frac{\ell^{3}}{l_{5}^{3}}
$$

The condition $l_{\mathrm{uv}} \simeq \ell$ then simplifies this expression to a relation between the four- and five-dimensional Planck 
scales, $\ell \simeq l_{5}^{3} / l 4^{2}$. This is precisely the dependence that arises from a direct Kaluza-Klein reduction from five to four dimensions for a horospherical brane embedded in $\mathrm{AdS}_{5}$ [7, 36.

The scale $\ell$ also represents the scale below which the $\mathrm{AdS}_{5}$ curvature becomes negligible and gravity becomes effectively five-dimensional. In this context, the correspondence (18) implies that the minimum measureable length can be identified with the scale at which fourdimensional physics breaks down. A similar conclusion was arrived at in a different context by a direct investigation of quantum systems with one extra dimension compactified on a circle [37].

Before concluding, we should discuss the validity of the assumptions we have made. Firstly, the GUP we have invoked in Eq. (6) is heuristic, in the sense that it has not been derived from first principles. Thus, although it ultimately leads to the trace-anomaly Friedmann equation, it is probable that the thermodynamic approach we have developed does not incorporate all the corrections that are expected to arise to the gravitational action. In particular, one would expect graviton loops to generate higher-order corrections that also lead to further $H^{4}$-correction terms in the Friedmann equation. Thus, our derivation is necessarily incomplete.

Another key assumption we made was that the apparent horizon should vary sufficiently slowly with respect to cosmic time, such that its area remains effectively constant during the time interval it takes for a quantum particle to be emitted. This is equivalent to assuming a quasi-de Sitter (inflationary) expansion and can be realised by a slowly-rolling, self-interacting scalar field (as we implicitly assumed). Such a field can be interpreted as the perfect fluid responsible for the energy-momentum flux through the horizon, Eq. (15). (A self-interacting scalar field minimally coupled to Einstein gravity is dynamically equivalent to a perfect fluid in a spatially isotropic universe.) On the other hand, a posteriori such an assumption is not necessary, since the thermodynamic Friedmann equation (17) admits solutions of the form

$$
H^{2}=\frac{1}{\alpha^{2} l_{4}^{2}}\left[1+\epsilon \sqrt{1-\frac{16 \pi l_{4}^{4} \alpha^{2}}{3} \rho}\right]
$$

where $\epsilon= \pm 1$. The $\epsilon=+1$ root is essentially the Starobinsky 38] model of inflation driven by an $R^{2}$ correction to the Einstein-Hilbert action, where $R$ is the Ricci curvature scalar [39]. In this case, one may regard the fluid crossing the apparent horizon as the conformal (massless) matter fields.

In conclusion, we have considered the effect of the generalized uncertainty principle on the entropy of the apparent horizon and found that the trace-anomaly Friedmann equation can be derived from the first law of thermodynamics when Eq. (18) is satisfied. For a generic CFT, such a correspondence implies that perturbative theory should break down at a scale $l_{\mathrm{uv}} \gtrsim \sqrt{n} l 4$ in a theory containing $\mathcal{O}(n)$ light species. For a CFT with a holographic dual, it identifies the minimum measureable length in four dimensions as the scale where physics becomes effectively five-dimensional. It is also worth remarking that trace-anomaly inflation provides an excellent fit to the Planck satellite observations of the cosmic microwave background anisotropies [40].
[1] M. J. Duff, Class. Quant. Grav. 11, 1387 (1994) arXiv:hep-th/9308075.

[2] M. Henningson and K. Skenderis, JHEP 9807, 023 (1998) arXiv:hep-th/9806087.

[3] J. Maldacena, Adv. Theor. Math. Phys. 2, 231 (1998) arXiv:hep-th/9711200.

[4] S. S. Gubser, I. R. Klebanov, and A. M. Polyakov, Phys. Lett. B 428, 105 (1998) arXiv:hep-th/9802109.

[5] E. Witten, Adv. Theor. Math. Phys. 2, 253 (1998) [arXiv: hep-th/9802150.

[6] O. Aharony, S. S. Gubser, J. Maldacena, H. Ooguri, and Y. Oz, Phys. Rept. 323, 183 (2000) arXiv:hep-th/9905111.

[7] S. S. Gubser, Phys. Rev. D 63, 084017 (2001) arXiv:hep-th/9912001.

[8] S. de Haro, S. N. Solodukhin, and K. Skenderis, Comm. Math. Phys. 217, 595 (2001) arXiv:hep-th/0002230; Class. Quant. Grav. 19, 5849 (2002) arXiv:hep-th/0209067.

[9] G. Compere and D. Marolf, Class. Quant. Grav. 25, 195014 (2008) arXiv:0805.1902.

[10] P. S. Apostolopoulos, G. Siopsis, and N. Tetradis, Phys. Rev. Lett. 102, 151301 (2009) arXiv:0809.3505.
[11] M. V. Fishetti, J. B. Hartle, and B. L. Hu, Phys. Rev. D 20, 1757 (1979); S. W. Hawking, T. Hertog, and H. S. Reall, Phys. Rev. D 63, 083504 (2001) arXiv:hep-th/0010232.

[12] J. D. Bekenstein, Phys. Rev. D 7, 2333 (1973).

[13] J. M. Bardeen, B. Carter, and S. W. Hawking, Comm. Math. Phys. 31, 161 (1973).

[14] S. W. Hawking, Phys. Rev. Lett. 26, 1344 (1971).

[15] W. Israel, Phys. Rev. Lett. 57, 397 (1971).

[16] J. D. Bekenstein, Phys. Rev. D 9, 3292 (1974).

[17] S. W. Hawking, Nat. 248, 30 (1974); Comm. Math. Phys. 43, 199 (1975).

[18] T. Jacobson, Phys. Rev. Lett. 75, 1260 (1995) arXiv:gr-qc/9504004.

[19] R. G. Cai and S. P. Kim, JHEP 0502, 050 (2005) arXiv:hep-th/0501055.

[20] M. Maggiore, Phys. Rev. D 49, 5182 (1994) arXiv:hep-th/9305163; Phys. Lett. B 319, 83 (1993) arXiv:hep-th/9309034; A. Kempf, G. Mangano, and R. B. Mann, Phys. Rev. D 52, 1108 (1995) arXiv:hep-th/9412167.

[21] D. Amati, M. Ciafaloni, and G. Veneziano, Phys. Lett. B 216, 41 (1989); Nucl. Phys. B 403, 707 (1993); K. 
Konishi, G. Paffuti, and P. Provero, Phys. Lett. B 234, 276 (1990).

[22] M. Maggiore, Phys. Lett. B 304, 65 (1993) arXiv:hep-th/9301067; F. Scardigli, Phys. Lett. B 452, 39 (1999).

[23] L. J. Garay, Int. J. Mod. Phys. A 10, 145 (1995) arXiv:gr-qc/9403008.

[24] A. J. M. Medved and E. C. Vagenas, Phys. Rev. D 70, 124021 (2004) arXiv:hep-th/0411022.

[25] M. Park, Phys. Lett. B 659, $698 \quad$ (2008) arXiv:0709.2307.

[26] C. M. Misner and D. H. Sharp, Phys. Rev. 136, B571 (1964).

[27] C. Adami, arXiv:quant-ph/0405005.

[28] R. Cai, L. Cao, and Y. Hu, Class. Quant. Grav. 26, 155018 (2009) arXiv:0809.1554.

[29] R. Cai, L. Cao, and Y. Hu, JHEP 0808, 090 (2008) arXiv:0807.1232.

[30] J. E. Lidsey, Class. Quant. Grav. 26, 147001 (2009) arXiv:0812.2791.

[31] D. V. Fursaev, Phys. Rev. D 51, 5352 (1995) arXiv:hep-th/9412161]; S. N. Solodukhin, Phys. Rev. D 57, 2410 (1998) arXiv:hep-th/9701106]; K. Srinivasan and T. Padmanabhan, Phys. Rev. D 60, 024007 (1999) arXiv:gr-qc/9812028; R. Banerjee and B. R. Majhi, JHEP 0806, 095 (2008) arXiv:0805.2220.
[32] G. Dvali and G. Gabadadze, Phys. Rev. D 63, 065007 (2001) arXiv:hep-th/0008054; G. Veneziano, JHEP 0206, 051 (2002) arXiv:hep-th/0110129.

[33] G. Dvali, arXiv:0706.2050 G. Dvali and M. Redi, Phys. Rev. D 77, 045027 (2008) arXiv:0710.4344]; G. Dvali and D. Lust, JHEP 0806, 047 (2008) arXiv:0801.1287]; G. Dvali, arXiv:0806.3801 G. Dvali and S. N. Solodukhin, arXiv:0806.3976 R. Brustein, G. Dvali, and G. Veneziano, JHEP 0910, 085 (2009) arXiv:0907.5516.

[34] G. Dvali and C. Gomez, Phys. Lett. B 674, 303 (2009) arXiv:0812.1940; G. Dvali and C. Gomez, arXiv:0907.3237

[35] L. Susskind and E. Witten, arXiv:hep-th/9805114.

[36] L. Randall and R. Sundrum, Phys. Rev. Lett. 83, 4690 (1999) arXiv:hep-th/9906064.

[37] B. Mu, H. Wu, and H. Yang, arXiv:0909.3635.

[38] A. A. Starobinsky, Phys. Lett. 91B, 99 (1980).

[39] S. W. Hawking, T. Hertog, and H. S. Reall, Phys. Rev. D 62, 0435012000 arXiv:hep-th/0003052; S. Nojiri and S. D. Odintsov, Phys. Lett. B 484, 119 (2000) arXiv:hep-th/0004097; S. Nojiri and S. D. Odintsov, Int. J. Mod. Phys. A 16, 3273 (2001) arXiv:hep-th/0011115]; E. Kiritsis, JCAP 0510, 014 (2005) arXiv:hep-th/0504219.

[40] P. A. R. Ade, et al., arXiv:1303.5082. 